\title{
Evaluation Methodology of the Refugees Movement Based on Factor-determining Model
}

\author{
Wenjie Li \\ School of North China Electric Power University, Baoding 071000, China \\ 363378037@qq.com
}

Keywords: Refugee Crises, Factor-Determining, Crises Index, Evaluating Safety and Efficiency

\begin{abstract}
In this paper, the generic factor-determining model is set up to construct a comprehensive decision-making system, aiming at evaluating the safety and efficiency of the refugee's movement. The whole work is based on the calculation of the crises index (CI). The larger CI is, the more dangerous and inefficient the refugee movement will be. Three independent families are preliminarily obtained to set with respect to the refugee source, the moving process, and the destination, respectively, containing11 different factors, to measure the crises index (CI) in quantification. Each factor is transferred to a detailed parameter to support the calculation of the significant coefficient.
\end{abstract}

\section{Parameters}

Since factors are difficult to determine because the scope is too large, so it's significant to classify all of those factors with parameters and estimate their effects to carry out the effective method to determining factors and measuring refugee crises

\begin{tabular}{cl}
\hline Symbol & Definition \\
\hline$C H$ & The total coefficient measuring the refugees crises \\
$C P$ & Coefficients of $\mathrm{H}$ that influence the refugee crises \\
$C D$ & Coefficients of $\mathrm{P}$ that influence the refugee crises \\
$H a$ & Coefficients of $\mathrm{D}$ that influence the refugee crises \\
$H m$ & Mortality \\
$H w$ & The value of Annual freshwater withdrawal \\
$H h$ & The number of hostilities \\
$H p$ & The volume of petroleum \\
$\alpha_{i}(i=1,2 \ldots 5)$ & Coefficients of each parameter \\
$P d$ & Distance between countries \\
$P C$ & The number of countries in the route \\
$P t$ & Valuation of different means of transportation \\
$\beta_{j}(j=1,2,3)$ & Coefficients of each parameter \\
$A r e a$ & The total area of the developed country \\
$L_{P}$ & The number of local population \\
$s_{j}$ & Criterion of average area per local person \\
$s_{2}$ & Criterion of average area per external person \\
$D P$ & Volume of ports, airports and railway stations \\
$D s$ & Area provide with asylums \\
$D r$ & The ratio of investment in the refugees \\
$\gamma_{k}(k=1,2,3)$ & Coefficients of each parameter \\
\hline &
\end{tabular}

\section{Establishment of the Model}

\subsection{Determine independent families}

It must be pointed out that the three families are independent and irrelative, and each of them produces a coefficient.

$\mathbf{H}$ (Home): Factors in this family derive from the analysis of the Middle East countries themselves' situation, seen as a driving force to refugees, as well as the individuals themselves.

$\mathbf{P}$ (Process): Factors in this family derive from the analysis of the process of refugees' movement. Take all conditions of move into account while deciding factors. 
D (Destination): Factors in this family derive from the situation analysis of the EU countries that would take in refugees, which determine the entry points.

\subsection{Measure refugee crises via coefficients of generic families}

Via analysis, it can be concluded that all of the three families are positive to the crises, so the effects of each family can add up fuzzily. Then the equation of the coefficient measuring the Refugees Crises is determined:

$C I=C H+C P+C D$

Where $C I$ is the total coefficient measuring the Refugees Crises, $C H, C P$ and $C D$ stand for coefficients of $H, P$, and $D$ that influence the refugee crises.

\subsection{Filtrating family members to obtain determinant factors}

Factors determined in $\mathbf{H}$ family:

1. Refugee's age

Peoples' age can decide their ability to move.

2. Health condition

Health of individuals can decide whether they move, so it can be included in the factors of $\mathrm{H}$. To measure health conditioned, we determine mortality to be a parameter.

3 warfare factors

Warfare is seen as a factor of $\mathrm{H}$ because the wars significantly influence the explosion of refugee crises.

4. Economic level of refugee countries

Factors determined in $\mathbf{P}$ family:

\section{Length of routes 2.Direction change 3. Transportation}

\section{Factors determined in D family:}

1. Situation of entries

2. EU countries' asylums capacity

If the destination country has enough shelters, it can provide refugees with asylums.

The countries' asylums capacity is important to the destination of refugee movement, so we have to measure the capacity.

3. Available resources for refugee

\subsection{Determination of parameters that quantify factors}

\section{Parameters measuring factors in $\mathbf{H}$}

We determine parameters and obtain the results below:

1.Aging ratio measuring refugee's age

We decide aging ratio (the proportion of people over 60 or 65 to total population) to the parameter measuring this factor.

2. Mortality measuring health condition

3. Annual freshwater withdrawals and number of hostilities measuring warfare

We have known that the wars mainly attribute to water sacristy and relationship with neighbors, so we use annual freshwater withdrawals and number of hostilities as parameters deciding this factor.

4. Volume of petroleum measuring economic level

Then we can use parameters above to calculate $\mathrm{CH}$ :

$$
\mathrm{CH}=\alpha_{1} \bullet \mathrm{Ha}+\alpha_{2} \bullet H \mathrm{~m}+\alpha_{3} \bullet H w+\alpha_{4} \bullet H h+\alpha_{5} \bullet H p
$$

Where $\mathrm{Ha}$ is the value of aging ratio, $\mathrm{Hm}$ stands for mortality, $\mathrm{Hw}$ means the value of Annual freshwater withdrawal, $H h$ is number of hostilities and $H p$ stands for the volume of petroleum. $\alpha_{i}(i=1,2 \ldots .5)$ mean coefficients of each parameter.

\section{Parameters measuring factors in $\mathbf{P}$}

1. Distance between countries measuring length of routes

2. Number of countries in the route measuring the direction change

3. Valuation of different means of transportation 
With consideration of safety and efficiency, we can assign values to each means. Then the initial scores (range 0 to 1 ) based on analysis can be determined: air transportation gets 0.5 , sea transportation gets 1 and land transportation gets 1 , too. And then, we can use parameters above to calculate $C P$ :

$$
C P=\beta_{1} \bullet P d+\beta_{2} \bullet P c+\beta_{3} \bullet P t
$$

Where $P d$ is the distance between countries, $P c$ means number of countries in the route, $P t$ is valuation of different means of transportation. $\beta_{j}(j=1,2,3)$ mean coefficients of each parameter.

\section{Parameters measuring factors in D}

1. Volume of ports, airports and railway stations measuring situation of entries

2. Area provide with asylums measuring EU countries' asylums capacity

The parameter is named as $D s$ :

$$
D s=\frac{\text { Area }-s_{1} \cdot L p}{s_{2}}
$$

Where Area is the total area of the developed country, $L p$ means the number of local population, $s_{1}$ and $s_{2}$ stand for criterion of average area per local/external person.

3 . The ratio of investment in the refugees measuring available resources for refugees. Finally we can use parameters above to calculate $C D$ :

$C D=\gamma_{1} D p+\gamma_{2} D s+\gamma_{3} D r$

Where $D p$ stands for Volume of ports, airports and railway stations, $D s$ means area provide with asylums, $D r$ is the ratio of investment in the refugees. $\gamma_{k}(k=1,2,3)$ mean coefficients of each parameter.

\subsection{Calculation of total CI based on parameters deriving from factors}

Putting (2)-(5) together into (1), we are able to calculate the coefficient of refugee crises $(C I)$ :

$$
C I=\sum \alpha_{i} \cdot H(\text { parameter })+\sum \beta_{j} \cdot P(\text { parameter })+\sum \gamma_{k} D(\text { parameter })
$$

This algorithm and previous method to finding determinant factors can be viewed in the block diagram in the followed figure.

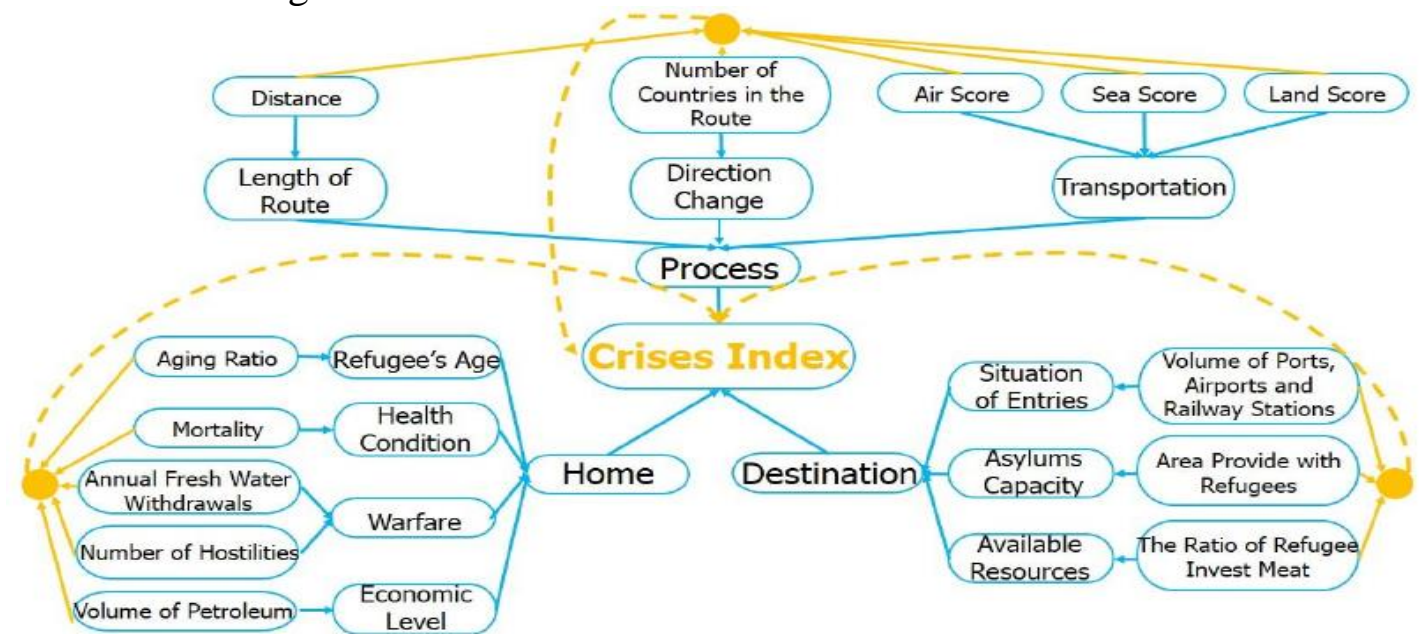

Fig1. Process of factors determination and Crises Index calculation

\section{Conclusion}

Through classification method which can be used to classify all the factors into three irrelative families, each determinant factor is finally confirmed to evaluate the safety and efficiency of refugee movement. Besides, the reasonable factors are quantified with measurable parameters. Then the refugee crises which called crises index can be measured, via which the extent of safety and efficiency are intuitively expressed. 


\section{References}

[1]. Salehin,S.; HuaichenZhang; Larriba,T.;Papakokkinos, G.; Bowler, E.; van Kasteren, J.M.N., "Designing of an Emergency Energy Module for relief and refugee camp situations: Case study for a refugee camp in Chad-Sudan border," in Sustainable Technologies (WCST), 2011 World Congress on , vol., no., pp.9-14, 7-10 Nov. 2011

[2]. Anderson,J.; Chaturvedi, A.; Lengacher, D.; Cibulskis, M., "Modeling the Health of Refugee Camps: An Agent-Based Computational Approach," in Computer-Based Medical Systems, 2006. CBMS2006. 19th IEEE International Symposium on , vol., no., pp.641-645, 0-0 0doi: 10.1109/CBMS.2006.118

[3]. Ito T., Umise S., Hoshi H., Suzuki M., Tani S., "Gait measurement and evaluation system for diagnosis of elderly people's gait condition to prevent fall," in Advanced Intelligent Mechatronics (AIM), 2015 IEEEInternational Conference on, vol.,no., pp.687-693, 7-11 July 2015 